

\title{
Enhancing Real-Time Delivery in Wireless Sensor Networks With Two-Hop Information
}

Yanjun Li, Chung Shue Chen, Ye-Qiong Song, Zhi Wang, Youxian Sun

\section{To cite this version:}

Yanjun Li, Chung Shue Chen, Ye-Qiong Song, Zhi Wang, Youxian Sun. Enhancing Real-Time Delivery in Wireless Sensor Networks With Two-Hop Information. IEEE Transactions on Industrial Informatics, 2009, 5 (2), pp.113-122. 10.1109/TII.2009.2017938 . inria-00431252

\section{HAL Id: inria-00431252 \\ https://hal.inria.fr/inria-00431252}

Submitted on 11 Nov 2009

HAL is a multi-disciplinary open access archive for the deposit and dissemination of scientific research documents, whether they are published or not. The documents may come from teaching and research institutions in France or abroad, or from public or private research centers.
L'archive ouverte pluridisciplinaire HAL, est destinée au dépôt et à la diffusion de documents scientifiques de niveau recherche, publiés ou non, émanant des établissements d'enseignement et de recherche français ou étrangers, des laboratoires publics ou privés. 


\title{
Enhancing Real-Time Delivery in Wireless Sensor Networks With Two-Hop Information
}

\author{
Yanjun Li, Chung Shue Chen, Member, IEEE, Ye-Qiong Song, Zhi Wang, Member, IEEE, and Youxian Sun
}

\begin{abstract}
A two-hop neighborhood information-based routing protocol is proposed for real-time wireless sensor networks. The approach of mapping packet deadline to a velocity is adopted as that in SPEED; however, our routing decision is made based on the novel two-hop velocity integrated with energy balancing mechanism. Initiative drop control is embedded to enhance energy utilization efficiency, while reducing packet deadline miss ratio. Simulation and comparison show that the new protocol has led to lower packet deadline miss ratio and higher energy efficiency than two existing popular schemes. The result has also indicated a promising direction in supporting real-time quality-of-service for wireless sensor networks.
\end{abstract}

Index Terms-Deadline miss ratio, energy utilization efficiency, quality-of-service (QoS), real-time, two-hop information, wireless sensor networks (WSNs).

\section{INTRODUCTION}

W IRELESS SENSOR NETWORKS (WSNs) have recently received increasing attention in the industrial communication community. A vivid vision of WSN can be described by the concept of "smart dust" [1]: small and cheap sensor nodes are embedded to sense the surroundings, communicate wirelessly, perform collaborative signal processing and make the environment intelligent. With WSN, it is possible to collect more real-time data than before, from places which are hazardous or inaccessible by wired technology. WSN can be used in many ways in industrial and factory automation [2]. For example, vibration, pressure or thermal sensors can be equipped to rotating machinery or conveyer belts to monitor their health. This helps to detect possible system failure and to trigger a preventive maintenance routine before a more costly repair is needed. WSNs are also useful for tracking leakage

Manuscript received September 29, 2008; revised January 11, 2009 and March 03, 2009. First published April 28, 2009; current version published May 06, 2009. This work was supported by the National Natural Science Foundation of China under Grant 60773181 and Grant 60873223, and in part by the National High-Tech Research and Development Plan of China under Grant 2006AA01Z218. Paper no. TII-08-09-0115.R2.

Y. Li is with the State Key Laboratory of Industrial Control Technology, Hangzhou 310027, China and LORIA-Nancy University, TRIO, Vandoeuvrelès-Nancy, France (e-mail: yjli.iipc@gmail.com).

C. S. Chen was with the Department of Probability and Stochastic Networks (PNA2), Centrum Wiskunde and Informatica, 1098 XG Amsterdam, The Netherlands (e-mail: cschen@ieee.org). This work was carried out during the tenure of an ERCIM "Alain Bensoussan" Fellowship Program.

Y.-Q. Song is with LORIA-Nancy University, TRIO, Vandoeuvre-lès-Nancy, France (e-mail: song@ @loria.fr).

Z. Wang and Y. Sun are with the State Key Laboratory of Industrial Control Technology, Hangzhou 310027, China (e-mail: wangzhi@iipc.zju.edu.cn; yxsun@iipc.zju.edu.cn).

Color versions of one or more of the figures in this paper are available online at http://ieeexplore.ieee.org.

Digital Object Identifier 10.1109/TII.2009.2017938 or radiation in chemical plants. Different from some existing best-effort services which may not have stringent packet timeliness requirement and can tolerate a significant amount of packet loss, these real-time industrial applications are much more demanding [3]. Out-of-date data are usually irrelevant and may even lead to negative effects to the system monitoring and control.

In industrial WSNs, traffic is dominated by readings and commands exchanged between sensors/actuators and control units. Providing quality-of-service (QoS) in such a scenario is to enable transmissions of periodic or sporadic messages within predefined deadlines in a reliable fashion; timeliness is especially important for crucial alarm messages. Since the wireless channel is random and time-varying, conventional deterministic QoS measures should be replaced by probabilistic ones. An important performance measure is the deadline miss ratio (DMR) which is defined as the ratio of messages that cannot meet deadlines [4]. Moreover, sensor nodes usually use battery for energy supply. Hence, energy efficiency is also an important design goal. It is usually defined by the energy consumed per successfully transmitted packet. Furthermore, in order to avoid network topology holes and achieve a longer network lifetime, node load and energy balance need to be considered.

Generally speaking, supporting real-time QoS in WSN can be addressed from different layers and mechanisms [5]. For example, medium access control (MAC) can offer channel access (one-hop) delay guarantee, while routing protocol in the network layer can support multihop QoS. Transmission scheduling can be used to provide conflict-free channel sharing based on regular network topology (e.g., tree, hexagonal layout, etc.) with techniques of topology control and clock synchronization [6]. Deterministic service delay bound for real-time applications is expected. In-network data aggregation is known as a good complement to routing protocols in reducing data redundancy and alleviating network congestion. Cross-layer optimization can provide further improvement. Among the above, without loss of generality, routing protocol has always played a crucial role in supporting end-to-end QoS. Here, we will focus on this domain and the design in this paper is oriented to more demanding applications which emphasize packet delivery timeliness and end-to-end QoS, e.g., alarm messages should be transmitted from sensor nodes to control center in time so as to take prompt actions. Energy efficiency and load balance are also among the design goals.

It is known from the literature [3] that for system simplicity most existing routing protocols are based on one-hop neighborhood information. However, it is expected that multihop information can lead to better performance in many issues 
including routing, message broadcasting, and channel access scheduling [7]-[10]. For computing two-hop neighborhood information in wireless ad hoc and sensor networks, some distributed algorithms and efficient information exchange schemes are reported in [10] and [11]. In a network of $n$ nodes, computing one-hop neighbors with $O(n)$ messages is trivial while computing two-hop neighbors seems to increase the complexity and overheads. However, a complexity analysis reported in [11] has shown that every node can obtain the knowledge of two-hop neighborhood by a total of $O(n)$ messages, each of $O(\log n)$ bits, which could be enough to address the ID and geographic position of nodes.

It is very likely that a system can perform better if more information is available and effectively utilized. By the study of asymptotic performance of a generic routing with multihop routing information [7], it is observed that the number of hops required from the source to sink decreases significantly from one-hop to two-hop information-based routing. However, the further gain from two-hop-based decision to three-hop-based decision is less attractive, especially if complexity increase is also a concern. In this paper, we propose a two-hop information-based real-time routing protocol and show its improvement over one-hop-based protocol SPEED [12]. The choice of two hops is a tradeoff between performance improvement and the complexity cost. The idea of two-hop routing is straightforward but how to use or integrate the information effectively so as to improve energy and real-time performance is generally nontrivial. The resulting design has the following novel features.

1) Compared with existing protocols that utilize only one-hop neighborhood information, it achieves lower deadline miss ratio and also higher energy efficiency.

2) A mechanism is embedded which can release nodes that are frequently chosen as packet forwarder. An improvement of energy balance throughout the network is achieved.

3) The simulation is built on Mica2-based [13] lossy link model, energy model and CSMA/CA MAC setting (similar to B-MAC [14]) which are very close to real systems.

The rest of this paper is organized as follows. Section II discusses related routing protocols for real-time QoS in WSN and explains the motivations. Section III presents our design. The performance of proposed protocol is reported in Section IV. Simulations and comparisons have shown its effectiveness. In Section V, we discuss possible enhancement. Finally, Section VI concludes this paper.

\section{REAL-Time Routing PROTOCOLS FOR WSN}

Generally speaking, there are three classes of routing policies that favor end-to-end delay performance guarantee in WSN: (i) tree-based routing; (ii) optimal routing based on shortestpath-first (SPF) principle by the knowledge of whole network topology; and (iii) geographic routing by the knowledge of node position.

Tree-based routing is popular in industrial WSN setting. ZigBee has provided a hierarchical tree routing scheme in which packets travel along the edges of the tree network. This approach suits the many-to-one traffic model and does not need routing table. End-to-end QoS (delay, energy consumption, etc.) can be estimated by the depth of the tree. However, the hierarchical tree routing can be very inefficient when two nodes in different branches but mutual radio range want to communicate with each other since packets must travel through the ZigBee coordinators. Ad hoc on-demand distance vector (AODV) routing is thus suggested as a supplement in this case. As proposed in [15], another solution is to look up the neighbor table in routing decisions so as to avoid long path and thus shorten the worst-case delay. Another drawback of tree-based routing is the problem of node energy consumption balancing. Nodes near the root of the tree will consume much more energy than the other and consequently lead to network topology holes.

A tree routing protocol is often not optimal as it does not choose the shortest path. AODV is one of the optimal routingbased protocols by SPF principle. However, additional overhead (e.g., extra packet and energy consumption) will be introduced in order to maintain the routing table. AODV is a reactive routing which is more favorable when communication is required infrequently. The route discovery on demand adds additional latency to packet transmission. This has been investigated in [17] and an AODV variant is proposed after introducing a new routing metric in evaluating path efficiency which includes end-to-end delay and energy consumption. As a result, the network lifetime is prolonged and end-to-end delivery ratio is improved for real-time embedded systems.

Another QoS aware routing protocol is proposed in [16] for WSN. It finds multiple least-cost and energy-efficient paths by extended Dijkstra's algorithm and pick the path that can meet end-to-end delay requirement during the connection. In addition, a class-based queueing model is employed to serve both best-effort and real-time traffics. Their approach, however, does not consider the impact of channel access delay. Besides, the use of class-based priority queueing mechanism is too complicated and costly for resource limited sensor nodes.

Geographic routing is popular in WSN since it does not need to maintain routing table and consequently can reduce network energy consumption. Resulting algorithms are highly scalable [18]. However, geographic routing protocols are in general not optimal since most of them are based on one-hop decision. In addition, determining node position will introduce some overheads and energy consumption. Several solutions exist for finding coordinates, e.g., using global positioning system (GPS). Note that for resource-limited WSN, using GPS can be a problem as the required positioning chips will increase the price and energy consumption. This problem can be alleviated by using positioning chips only in some nodes, while other nodes calculate positions with the assistance of their neighbors. On the other hand, existing localization techniques such as triangulation, multilateration and diffusion [18] can provide GPS-free solutions. Some ranging techniques have also been specified in the IEEE 802.15.4a standard [19], e.g., estimating distance by measuring the difference of propagation delays.

In geographic routing, the heuristic greedy forwarding protocol SPEED [12] is the first one addressing real-time guarantees for WSN. Relay velocity toward a next-hop node is identified by dividing the distance progress by its estimated forwarding delay. Packet deadline is mapped to a velocity requirement. The node with the largest relay velocity higher than 
the velocity requirement is selected in the highest probability. If there is no neighbor node that can meet the requirement, the packet is dropped probabilistically to regulate network workload. Meanwhile, back-pressure packet rerouting in large-delay link is conducted to divert and reduce packets injected to a congested area. MM-SPEED [20] extends SPEED by defining multiple delivery velocities for packets with different deadlines in supporting different QoS. Real-time power-aware routing (RPAR) [21] is another variant of SPEED. A node will adaptively change its transmission power by the progress towards destination and packet's due time in order to meet the required velocity in the most energy-efficient way. Note that all the above protocols are based on one-hop neighborhood information.

In our proposed scheme, we also adopt the approach of mapping packet deadline to a velocity, which is known as a good metric to delay constrained packet delivery. However, our routing decision will be made based on two-hop neighborhood information and corresponding metrics. It is therefore named as two-hop velocity-based routing (THVR). Note that generally speaking it is also possible to employ other metrics, e.g., by packet lifetime or hop count, to design routing protocols. The idea of two-hop information-based routing is generic and applicable. Here, we will focus on THVR. The routing design and details are given in the next section.

\section{DESIGN OF THVR FOR RT-WSN}

Although two-hop information-based routing is intuitively helpful to improve the routing decision, an explicit mechanism is necessary. It is worth noting that THVR primarily aims at lowering packet DMR for demanding real-time WSN but will also consider energy utilization efficiency that has not been explicitly addressed in SPEED and MM-SPEED.

As assumed in most geographic routing algorithms, each node in the network is aware of the geographic location of itself and the destination, via GPS or other localization techniques [18], [19] as mentioned in Section II. The information can be further exchanged among two-hop neighbors [10], [22]. Thus, each node is aware of its immediate and two-hop neighbors, and their locations. This is achieved by two rounds of HELLO messages. First, each node informs its neighbors about its existence (ID, position, remaining energy, etc.). Next, each node sends message to all its neighbors informing about its one-hop neighbors. If the network is static or with low mobility, this could be done at one stroke until there is node failure. Otherwise, in a mobile network, each node periodically emits additional HELLO messages to maintain two-hop information. Too old entries are removed from the neighbor table, as corresponding nodes have moved out of one-hop or two-hop range.

To be detailed below, our design is mainly composed of three components: (i) forwarding metric; (ii) delay estimation and update; and (iii) initiative drop control.

\section{A. Forwarding Metric}

To begin with, some definitions are introduced. For each node, $i, \mathbf{N}(i)$ is used to denote the set of its one-hop neighbors. The source and destination nodes are labeled by $S$ and $D$, respectively. The distance between a pair of nodes $i$ and $j$

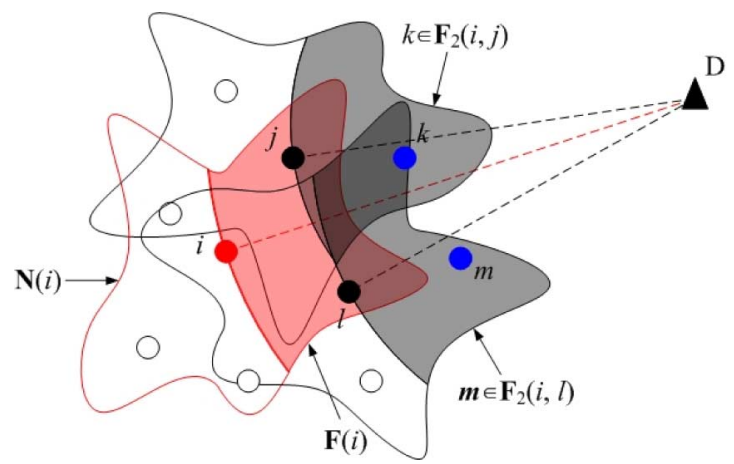

Fig. 1. Illustration of node's neighbor set, one-hop and two-hop forwarder set.

is denoted by $d(i, j)$. Consequently, the required end-to-end packet delivery velocity for deadline, $t_{\mathrm{set}}$, is defined as

$$
S_{\mathrm{set}}=\frac{d(S, D)}{t_{\mathrm{set}}} .
$$

$\mathbf{F}(i)$ is defined as the set of node $i$ 's potential forwarders which will make a progress towards the destination, i.e.,

$$
\mathbf{F}(i) \triangleq\{j \mid d(i, D)-d(j, D)>0, j \in \mathbf{N}(i)\} .
$$

$\mathbf{F}_{\mathbf{2}}(i, j)$ is defined to represent the set of corresponding two-hop potential forwarders, i.e.,

$$
\begin{aligned}
\mathbf{F}_{2}(i, j) \triangleq\{k \mid d(j, D)-d(k, D)> & 0, \\
& j \in \mathbf{F}(i), k \in \mathbf{N}(j)\} .
\end{aligned}
$$

An illustration of node's neighbor set, one-hop and two-hop forwarder set is shown in Fig. 1.

In SPEED, the core component SNGF (stateless nondeterministic geographic forwarding) works as follows. Upon receiving a packet, node $i$ calculates the velocity provided by each of the forwarding nodes in $\mathbf{F}(i)$, which is expressible as

$$
S_{i}^{j}=\frac{d(i, D)-d(j, D)}{\operatorname{Delay}_{i}^{j}}
$$

where $j \in \mathbf{F}(i)$ and Delay $_{i}^{j}$ denotes the estimated hop delay between $i$ and $j$. If there exists $j$ such that $S_{i}^{j} \geq S_{\text {set }}$, it will be chosen as the forwarder with probability $P(j)$ following the discrete exponential distribution below [12]:

$$
P(j)=\frac{\left(S_{i}^{j}\right)^{K}}{\sum_{j=1}^{N}\left(S_{i}^{j}\right)^{K}}
$$

where $N$ is the number of candidates in $\mathbf{F}(i)$ and $K$ is a weighting exponent to tradeoff between load balance and optimal delivery delay. A larger $K$ will lead to a shorter end-to-end delay, while a smaller one can achieve a better load balance.

In our proposed THVR, similarly to SPEED, by two-hop information, node $i$ will calculate the velocity provided by each of the two-hop forwarding pairs $\left\{\mathbf{F}(i), \mathbf{F}_{2}(i, j)\right\}$, i.e.,

$$
S_{i}^{j \rightarrow k}=\frac{d(i, D)-d(k, D)}{\operatorname{Delay}_{i}^{j}+\operatorname{Delay}_{j}^{k}}
$$


where $j \in \mathbf{F}(i)$ and $k \in \mathbf{F}_{2}(i, j)$. For node pair $(j, k)$ satisfying $S_{i}^{j \rightarrow k} \geq S_{\text {set }}$, we denote it by set $\mathbf{S}$. Beyond comparing the potential forwarding velocities, we also take into account node's remaining energy level, and thus define the following new joint metric:

$$
v e_{i}^{j \rightarrow k}=C \times \frac{S_{i}^{j \rightarrow k}}{\sum_{(j, k) \in \mathbf{S}} S_{i}^{j \rightarrow k}}+(1-C) \times \frac{E_{j} / E_{j}^{0}}{\sum_{j}\left(E_{j} / E_{j}^{0}\right)}
$$

where $E_{j}$ is the remaining energy of forwarder candidate $j$, while $E_{j}^{0}$ is its initial energy, and $C \in[0,1]$ is the weighting factor incorporating energy level into the joint metric. Note that larger $C$ tends to favor end-to-end delay performance, while smaller one can distribute traffics to nodes in higher energy level and result in a better energy balance. Clearly, a setting of $C$ relies on deadline requirements. The larger the deadline, the smaller $C$ could be.

By (7), the node in $\mathbf{F}(i)$ (e.g., node $j$ ) with the largest ve will be chosen as the forwarder. The routing then proceeds and the mechanism is repeated at the selected node iteratively. In THVR, the sender will search the largest velocity in two-hop neighborhood before making the forwarding decision. However, in SPEED [12], it is only one-hop optimized. For example, if there is a topology hole after the first forwarding node, SPEED will get a critical problem and have to activate back-pressure rerouting. By THVR, this kind of problems can be alleviated. Inherently, THVR has one-hop more prediction capability as using a "telescope" in finding the path. Generally speaking, even if the starting choice is not the globally optimized one, it may still have a better chance to gradually be corrected due to the farther sight and view.

\section{B. Delay Estimation}

From (6), it is observable that packet delay estimation from sender to its potential forwarder has played an important role in the velocity. In general, the delay of a packet from a node $i$ to its immediate forwarder $j$ is expressible as

$$
\text { Delay }_{i}^{j}=\left(\text { Delay }_{\mathrm{MAC}}+\text { Delay }_{\mathrm{TX}}\right) \times C_{i}^{j}
$$

where Delay ${ }_{\mathrm{MAC}}$, Delay ${ }_{\mathrm{TX}}$ and $C_{i}^{j}$ are used to represent the MAC delay, and transmission count, respectively. The transmission time includes the queueing delay (depending on the load of the node) and the packet transmission time (determined by the packet/ACK size and the bandwidth). The transmission count refers to the number of retransmissions involved since automatic repeat-request (ARQ) is adopted when packet fails to be transmitted due to collision or lossy link.

To have packet delay estimation in identifying (6), we adopt the method of window mean with exponentially weighted moving average (WMEWMA), which has been shown in [23] with its best estimation performance among existing techniques. The estimate of Delay ${ }_{i}^{j}$ for time instant $(t+1)$ is given by

$$
\operatorname{Delay}_{i}^{j}(t+1)=\alpha M_{i}^{j}(t)+\frac{1-\alpha}{T} \sum_{k=\max (1, t-T)}^{t-1} \operatorname{Delay}_{i}^{j}(k)
$$

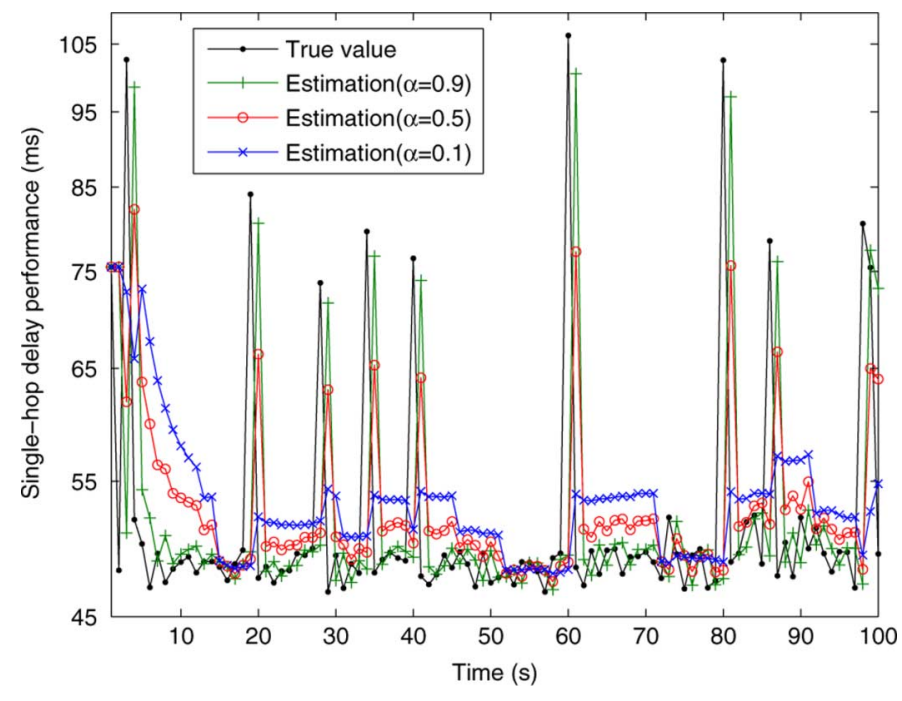

Fig. 2. Delay estimation performance under different values of $\alpha$.

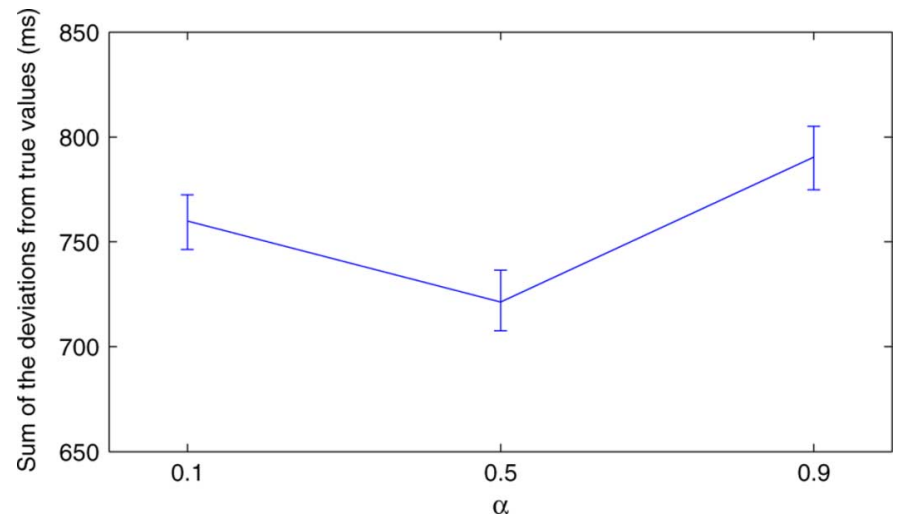

Fig. 3. Deviation from true values under different values of $\alpha$. The $90 \%$ confidence interval is also plotted.

where $T$ is the time window, $M_{i}^{j}(t)$ is the newly measured delay (known from the most recent packet), and $0<\alpha<1$ is the tunable weighting coefficient. It is clear that a large $\alpha$ will emphasize $M_{i}^{j}(t)$ and fits the case where delay variance is small, while a small $\alpha$ is more suitable if the variance is significant. A demonstration of the delay estimates under different $\alpha$ is plotted in Fig. 2, while the sum of deviations is indicated in Fig. 3. With a small $\alpha(\alpha=0.1)$, the delay estimate is insensitive and too slow to capture the system's immediate fluctuation and thus may result in a big deviation sum. However, when $\alpha$ is too large $(\alpha=0.9)$, the update to the delay estimate appears too rigorous while nearly ignoring the historic average and results in an even larger deviation sum. As indicated in Fig. 3, the deviation is the lowest when $\alpha$ is set to 0.5 which is quite robust generally. Note that it is also possible to design an adaptive tuning mechanism with reference to encountered delay variance. However, we will not go into the detail in this paper.

To identify the link delay of a packet, a sender will stamp the time when the packet is first sent and then compare it with the time when an acknowledgment (ACK) is received. On the other hand, to update the link delay information to other nodes in the routing path, after receiving the ACK with delay information from its forwarder, the node will initiate a feedback packet, which contains the updated delay of the forwarding link, to its 


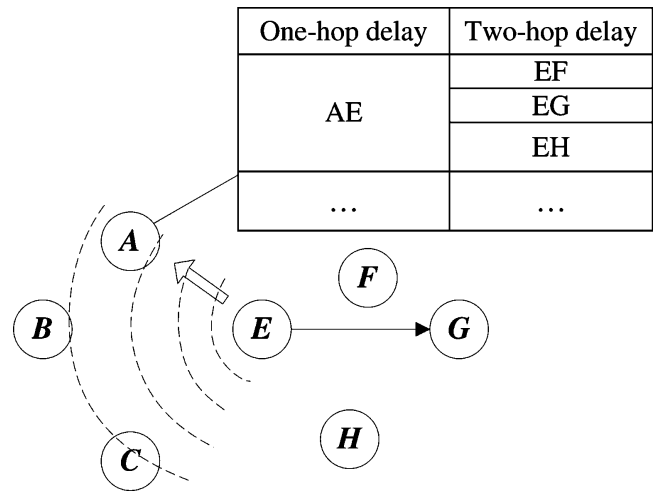

Fig. 4. An example of two-hop delay update.

parent node, i.e., the one who chose it as a forwarder. Meanwhile, other neighboring nodes which can overhear the feedback will also update their delay records. Fig. 4 shows an example of link delay update when node $G$ is chosen as the forwarder of node $E$. Delay ${ }_{E}^{G}$ is first updated at $E$ after receiving ACK from $G$, and then feedback to node $A$. Nodes $B$ and $C$ overhear the feedback. As a result, the delay field $E G$ in their records, e.g., a two-hop delay table, will be updated by the new information and (9). It should be noted that the two-hop information will enlarge the table of delay profile stored in each node. This needs to be considered if sensor nodes employed have very limited memory.

\section{Initiative Drop Control}

If no node in the two-hop forwarding set can provide the required velocity, the following initiative drop control will be conducted. To begin with, some technical details are defined. Let $e_{j}$ be the packet loss ratio of node $j(j \in \mathbf{F}(i))$ and $N$ be the number of nodes in $\mathbf{F}(i) .{ }^{1}$ We define the following forwarding probability of node $i$, denoted by $u_{i}$, as

$$
u_{i}= \begin{cases}1-K_{1} \frac{\sum_{j=1}^{N} e_{j}}{N}, & \frac{d(i, D)}{d(S, D)}>\frac{1}{2} \\ 1-K_{2} \frac{\sum_{j=1}^{N} e_{j}}{N}, & \frac{d(i, D)}{d(S, D)} \leq \frac{1}{2}\end{cases}
$$

where $K_{1}$ and $K_{2}$ are proportional gains with $K_{1}>K_{2}>$ $0,0 \leq u_{i} \leq 1$. The forwarding probability is jointly decided by the loss ratio in the forwarding set and the node position. First, the node that is close to the destination has higher forwarding probability. This is designed by the fact that a packet near the destination has already traveled a long way along the routing and many nodes have consumed energy to relay it, thus it is worthwhile to try more efforts and see whether we can finally deliver it successfully. Although the current hop may not be able to meet the required velocity, it is possible to meet the end-to-end requirement finally if the coming hops may have relatively short delays. However, if the packet is still at a node near the source that cannot meet the velocity, from the point-of-view of energy utilization efficiency, it will be more efficient to drop it earlier.

\footnotetext{
${ }^{1}$ Note that $e_{j}$ can be obtained by reading link quality indicator (LQI) field in some sensor nodes (e.g., MicaZ [13]).
}

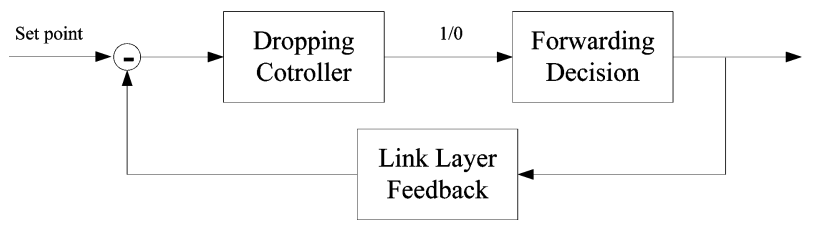

Fig. 5. Initiative drop control.

Second, by (10), the node whose forwarding candidates have lower average loss ratio has higher forwarding probability. As shown in Fig. 5, the link layer collects the node packet loss ratio and feeds it back to the dropping controller, which calculates the forwarding probability according to (10). For WSN, the broadcast nature of the wireless medium allows snooping on the channel. Losses can be known by tracking the link sequence number in the packets from each source. Various low-power listening mechanisms exist [23] that would enable snooping at a much lower cost. An alternative approach is to use received signal strength as an indication of link quality. Note that the controller is a proportional controller and the function of the control loop is to force the loss ratio of neighbors to converge to the set point, e.g., 0. The output of the controller is deterministic and binary. If the output of the controller is 1 , the node will forward the packet to the candidate that provides largest ve regardless of the velocity. Otherwise, dropping is made to maintain the delay requirement.

\section{PERformance Evaluation}

The proposed THVR is simulated in Prowler-Rmase [24], [25]. Prowler is a probabilistic wireless network simulator, capable of simulating wireless distributed systems, from the application to the physical communication layers. It provides simple yet realistic radio/MAC models based on the Berkeley mote platform, and supports an event-driven structure similar to TinyOS/NesC. Rmase has extended Prowler to more options of topologies, application models, and routing designs.

To be close to practical WSN and realistic implementation, we set the MAC layer, link quality model, and energy consumption parameters according to Mica2 Motes [13] with MPR400 $(915 \mathrm{MHz})$ radio. Nodes are distributed in a $200 \mathrm{~m} \times 200 \mathrm{~m}$ area following Poisson point process with node density $\rho=$ 0.005 node $/ \mathrm{m}^{2}$. This node density is chosen by the method described in [26] to ensure a high level of network connectivity. To simulate multihop transmissions with a large enough number of hop counts, we limit the source nodes to the left-lower corner of the above region, while the sink is fixed at the location $(200 \mathrm{~m}$, $200 \mathrm{~m}$ ). The size of the neighbor table for each node is set to 400 bytes for all the tested protocols, which is found sufficient to store neighbor information within two hops. Note that for THVR, relatively each node needs more memory to maintain two-hop information. Practically, the average number of neighbors within two hops is around 20 with average node degree 6 under our simulation settings. Theoretically, if we consider an ideal node deployment over a grid without loss of the generality, the number of $h$-hop neighbors of a node is given by $(2 h+1)^{2}-1$. Thus, the memory space complexity is polynomial with $O\left(h^{2}\right)$, which is acceptable in practice for $h=2$. 


\section{A. MAC Settings}

Following the default CSMA scheme (similar to B-MAC [14]) in Mica2 Motes, to initiate a packet transmission, a sensor node will generate a random initial waiting time uniformly distributed in the range of [200, 328] bit-time (by Mica2 Motes, one bit-time equals to $1 / 40000 \mathrm{~s}$ ) and start a timer. Upon timer expiration, the channel is sensed. If it is found idle, a packet is transmitted. Otherwise, it backoffs and then continues the sensing until the channel is found idle. The backoff time is uniformly distributed in $[100,130]$ bit-time. To improve delivery reliability, ARQ is employed here. If the total number of transmission count is greater than 7, the packet will be dropped. This is for avoiding excessive tries to a bad link or a too busy channel.

\section{B. Link Model}

We adopt the packet reception rate (PRR) model [27] for lossy WSN links. It is built on experimental measures of practical systems with respect to statistics of wireless channel. With the standard noncoherent FSK modulation and Manchester encoding, the PRR, $0 \leq p(s) \leq 1$, of a wireless link is expressible as

$$
p(s)=\left(1-\frac{1}{2} \exp \left(-\frac{\gamma(s)}{2} \frac{1}{0.64}\right)\right)^{8(2 f-l)}
$$

where $s$ is the transmitter-receiver distance, $\gamma(s)$ is the signal-to-noise ratio (SNR), and $f$ is the frame size which equals to 50 bytes including preamble $l$ ( 2 bytes), payload and CRC ( 2 bytes). Here, we adopt the setting of [27]. Note that the maximum packet size allowed is 241 bytes for Mica2. This model takes into account both distance-dependent path loss and log-normal shadowing in characterizing wireless links. For transmitting power $P_{t}$, the $\operatorname{SNR} \gamma(s)$ is expressible as

$$
\gamma(s)_{\mathrm{dB}}=P_{t \mathrm{~dB}}-P L(s)_{\mathrm{dB}}-P_{n \mathrm{~dB}}
$$

where, according to MICA2 radios, $P_{t \mathrm{~dB}}$ is set at $0 \mathrm{dBm}$, the noise floor $P_{n \mathrm{~dB}}$ is at $-115 \mathrm{dBm}$, and the path loss $\mathrm{PL}(s)_{\mathrm{dB}}$ is modeled as

$$
\mathrm{PL}(s)_{\mathrm{dB}}=\mathrm{PL}\left(s_{0}\right)_{\mathrm{dB}}+10 n \log _{10}\left(s / s_{0}\right)+X_{\sigma \mathrm{dB}}
$$

where $n$ is the path loss exponent, $s_{0}$ is the reference distance (1 meter), and $X_{\sigma}$ denotes the log-normal shadowing with zero mean and variance $\sigma^{2}$. In the coming simulations, we set $n=3$ and $\sigma=3$. For each transmission, a random number $x$ is generated and then compared with $p(s)$ as in a lossy WSN link. When $x \leq p(s)$, the packet is assumed successfully transmitted. Otherwise, it is considered lost and a retransmission will be initiated.

\section{Energy Model}

In WSN, the energy consumed in a node is mainly due to packet transmission $\left(E_{t x}\right)$, reception $\left(E_{r x}\right)$ and channel sensing $\left(E_{c s}\right)$ to check whether it is clear. The total energy consumed is thus expressible as

$$
\begin{aligned}
E & =E_{t x}+E_{r x}+E_{c s} \\
& =V \times\left(f \cdot I_{t x} T_{t x}+f \cdot I_{r x} T_{r x}+I_{c s} T_{c s}\right)
\end{aligned}
$$

TABLE I

Mica2 Motes-BASEd ENERgy Model [14]

\begin{tabular}{|l|c|c|}
\hline Operations & Duration $(\mathrm{ms})$ & Current $(\mathrm{mA})$ \\
\hline \hline Transmit 1 byte $(0 \mathrm{dBm}):$ & $0.416\left(T_{t x}\right)$ & $20\left(I_{t x}\right)$ \\
Receive 1 byte: & $0.416\left(T_{r x}\right)$ & $15\left(I_{r x}\right)$ \\
Channel Sensing: & $0.35\left(T_{c s}\right)$ & $15\left(I_{c s}\right)$ \\
\hline
\end{tabular}

where $f$ is the packet size (i.e., 50 bytes), $I_{t x}, I_{r x}$, and $I_{c s}$ denote the current required during transmission, reception and channel sensing, respectively, $V$ is the voltage supply (by default, $3 \mathrm{~V}$ ), and $T_{t x}, T_{r x}$, and $T_{c s}$ refer to the corresponding activity durations, as listed in Table I, [14].

\section{Simulation Results}

In this section, a detailed performance investigation of THVR is conducted and compared with SPEED [12] and the wellknown PRR-distance-product routing metric, $\mathrm{PRR} \times d$, proposed in [28], in which $d$ is the distance traversed towards destination, which was verified superior to simple greedy geographic routing.

In the first set of simulation, we consider there is one source node located at $(20 \mathrm{~m}, 20 \mathrm{~m})$, while the sink is at $(200 \mathrm{~m}, 200 \mathrm{~m})$. The source generates a CBR flow at 1 packet/s with packet frame size equal to 50 bytes (including preamble, payload, and CRC). The value of $C$ in (7) is set at 0.9 to emphasize end-to-end delay performance. In each run, 500 packets are transmitted. Fig. 6 shows the result under different deadline requirements ranging from 900 to 1800 ms. As shown in Fig. 6(a), it is clear that with an increase of deadline, the DMR of a protocol has decreased generally by the fact that more packets can finally be forwarded to the destination due to a longer allowable duration. As the deadline increases, their DMRs will converge to some corresponding levels. It can be observed that THVR has lower DMR than all the other in general. When deadline is stringent (e.g., less than $1200 \mathrm{~ms}$ ), the advantage is especially significant. Generally speaking, SPEED with $K=10$ has smaller DMR than that with $K=2$ as expected. On the other hand, the metric $\mathrm{PRR} \times d$ is known good at choosing a link for better reliability and routes in best effort. However, it lacks an explicit consideration of packet timeliness and delay performance. Clearly, compared with the other two protocols, the proposed THVR is able to enhance real-time delivery by an effective integration of two-hop information. It has inherently a higher capability in path finding.

Fig. 6(b) shows the energy consumed per packet successfully transmitted. The consumption has similar tendency and characteristic as that in DMR, Fig. 6(a). By a high tolerance of packet delay (e.g., deadline larger than $1400 \mathrm{~ms}$ ), DMR tends to be stable and the number of packets successfully transmitted from end to end is also quite stable. This supports the convergence of overall energy consumption. In comparing to the other protocols, Fig. 6(b) clearly shows that THVR is more energy efficient. One of the major reasons is that THVR has a better capability in forwarding packets to a small delay path. This has resulted in a smaller DMR, smaller retransmission rate, and also higher energy utilization efficiency. Besides, the initiative drop 


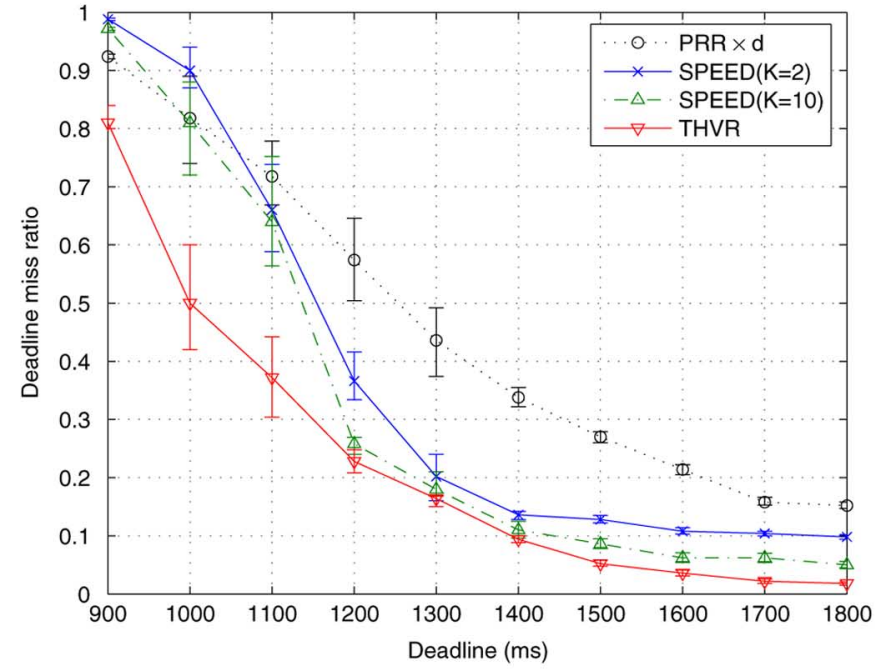

(a)

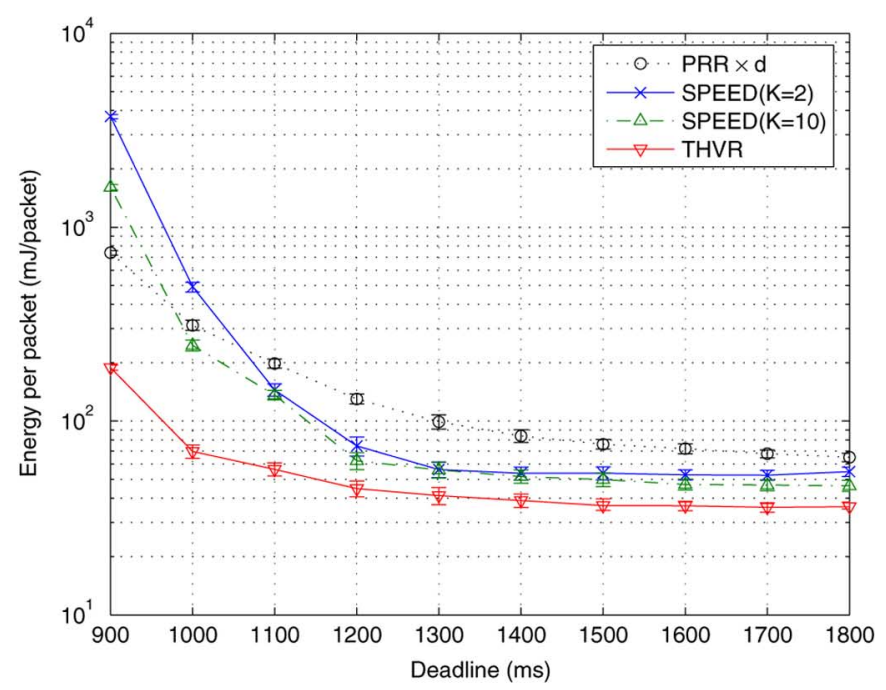

(b)

Fig. 6. Performance of: (i) PRR-distance-product routing; (ii) SPEED, with $K=2$ and 10 in (5), respectively; and (iii) THVR, each with 90\% confidence interval. (a) DMR with deadline varied. (b) Energy consumed per successfully transmitted packet.

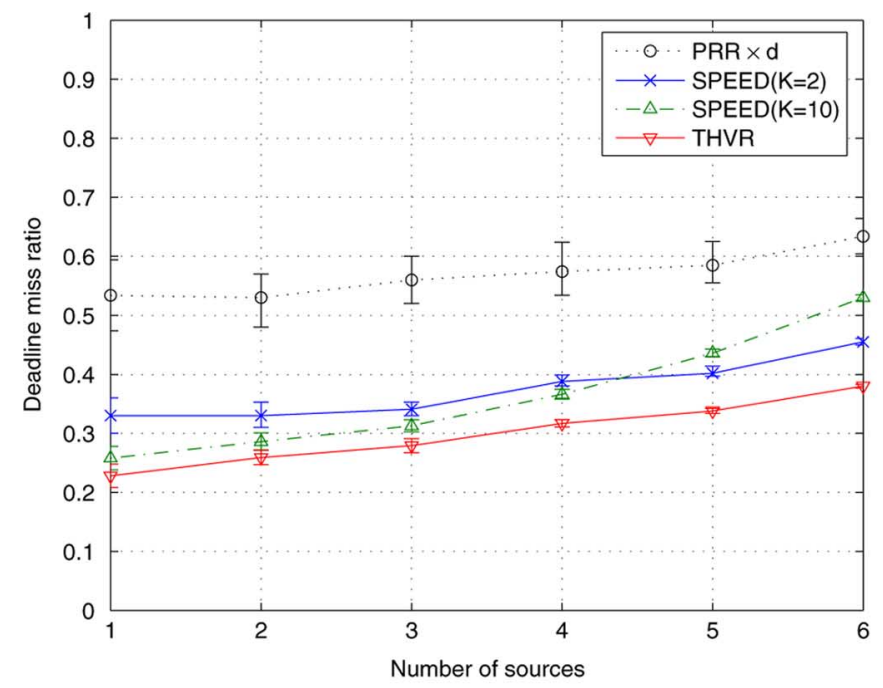

(a)

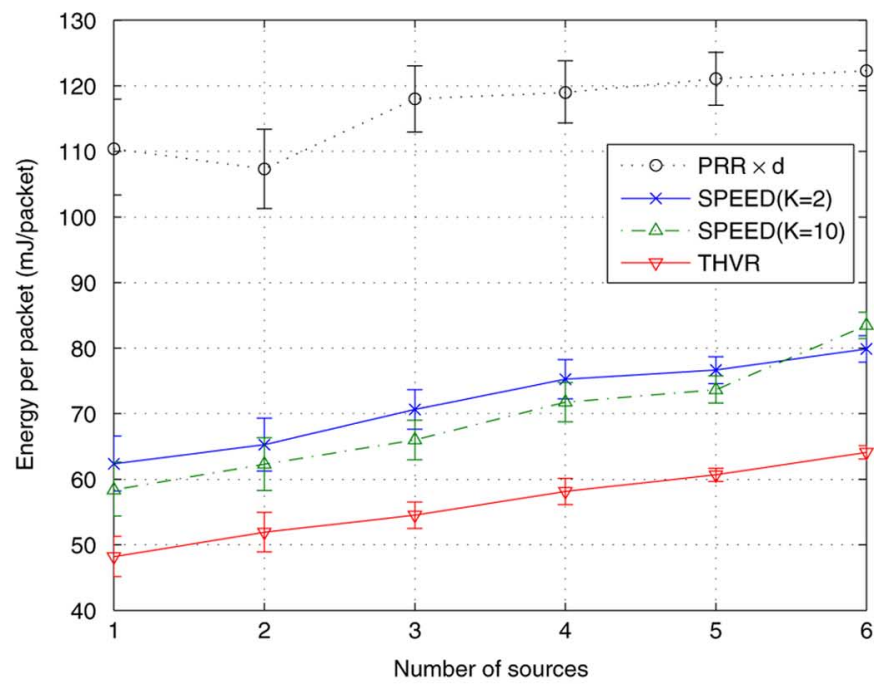

(b)

Fig. 7. Performance of: (i) $($ PRR $\times d$ )-routing; (ii) SPEED; and (iii) THVR, each with $90 \%$ confidence interval indicated, while the number of source nodes increases from 1 to 6 . (a) DMR with number of sources varied. (b) Energy consumed per successfully transmitted packet.

control has a positive effect in energy saving. As indicated by Fig. 6, THVR outperforms SPEED and also PRR $\times d$ geographic routing in both DMR and energy efficiency performance under the workload of single CBR flow.

Furthermore, we investigate the performance of THVR under different workload. Fig. 7(a) shows the DMR in which the number of sources increases from 1 to 6 . Each source generates a CBR flow at 1 packet/s, while the deadline requirement is fixed at $1200 \mathrm{~ms}$. The source nodes are located in the left bottom area, as highlighted in Fig. 8(a) and labeled with ID $\{190,116,1,112,93,158\}$, respectively. On the other hand, Fig. 7(b) shows the energy consumption performance of the three protocols. It is clear that as the number of sources increases, both the DMR and energy consumption generally increase. The increase in DMR is resulted by the increased channel busy probability, packet collisions at MAC, and network congestion, due to the increased number of sources and consequent traffics. The comparison indicates that THVR has lower DMR and also lower energy consumption per successfully transmitted packet, as shown in Fig. 7(a) and (b), respectively. This reflects the general improvement by THVR. It is worth pointing out that as the number of sources increases from 4 to 6 , SPEED with $K=2$ will outperform SPEED with $K=10$. This is due to the benefit of load balance with $K=2$ in the case the workload is heavy and traffic congestion is more likely to happen.

In addition, we conduct the following investigations to study the energy balance performance of the proposed cost function (7). First, deadline is relaxed to a large value of $2000 \mathrm{~ms}$ so that a larger end-to-end delay is allowable. The value of $C$ in THVR 


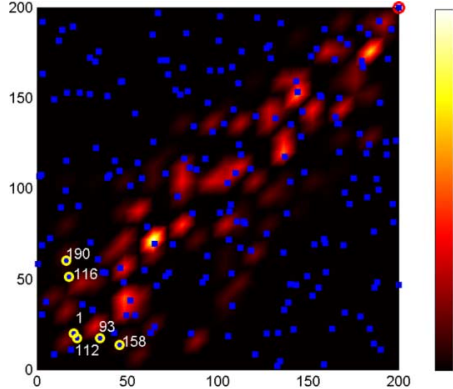

(a)

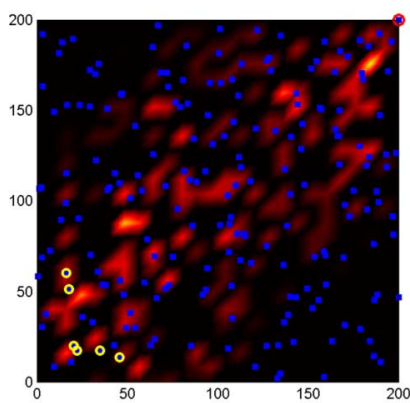

(c)

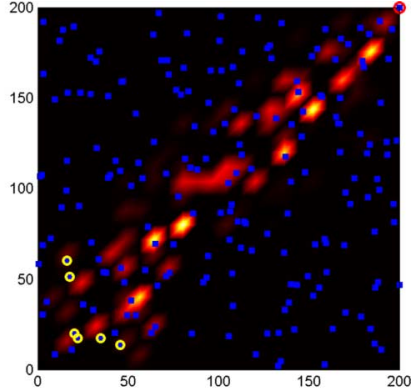

(b)

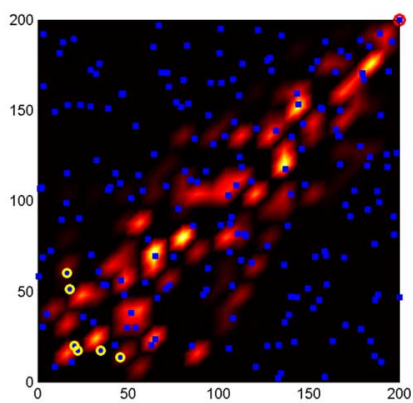

(d)
Fig. 8. Energy consumption distribution and magnitude in different protocols (a) $\operatorname{SPEED}(K=2)$. (b) $\operatorname{SPEED}(K=10)$. (c) $\operatorname{THVR}(C=0.7)$. (d) $\operatorname{PRR} \times$ d.

is set at 0.7 so as to have a larger weighting on the factor of residual energy in forwarder selection. Six source nodes are located in the left-bottom area as usual. Simulation stops when a total of 400 packets are successfully delivered within deadline by each source. Fig. 8 depicts the energy consumption distribution and magnitude of nodes in the WSN. In a comparison of Fig. 8(a) and (b), it is observable that SPEED with $K=10$ has energy consumption footprints more centralized along the diagonal path, while the setting of $K=2$ is able to spread the footprints to a wider area. Comparing the four distributions in Fig. 8, it is clear that THVR has the most even energy consumption that is shared among a large number of nodes. It can be expected that THVR will have a longer system lifetime due to the better balancing. On the other hand, their delay performance in the WSN is shown in Table II under the simulation. THVR has the lowest DMR and energy consumption per successfully transmitted packet. However, it is worth noting that the value of $C$ should be carefully chosen in the delay and load balance tradeoff. Otherwise, the end-to-end delay performance could be over-sacrificed, and consequently much degraded.

\section{Discussions}

Generally, an instant two-hop delay updating will induce more overheads than that required for one-hop information updating. This issue will impact our two-hop-based design as well. It can be observed from Fig. 4 that a further feedback will be sent from a child node to its parent node as aforementioned. We measure the total amount of overheads (ACK packets) encountered and plot it (labeled by "THVR_IU") in Fig. 9, and compare to that required in SPEED. In our case, it is nearly two
TABLE II

Deadline Miss Ratio and EnERgy Consumption Under Fig. 8

\begin{tabular}{|l|c|c|}
\hline Routing protocols & Deadline miss ratio & Energy per packet $(\mathrm{mJ} /$ packet $)$ \\
\hline \hline$P R R \times d$ & 0.3144 & 67.74 \\
SPEED $(K=2)$ & 0.1724 & 55.50 \\
SPEED $(K=10)$ & 0.2077 & 57.59 \\
THVR & 0.0967 & 54.49 \\
\hline
\end{tabular}

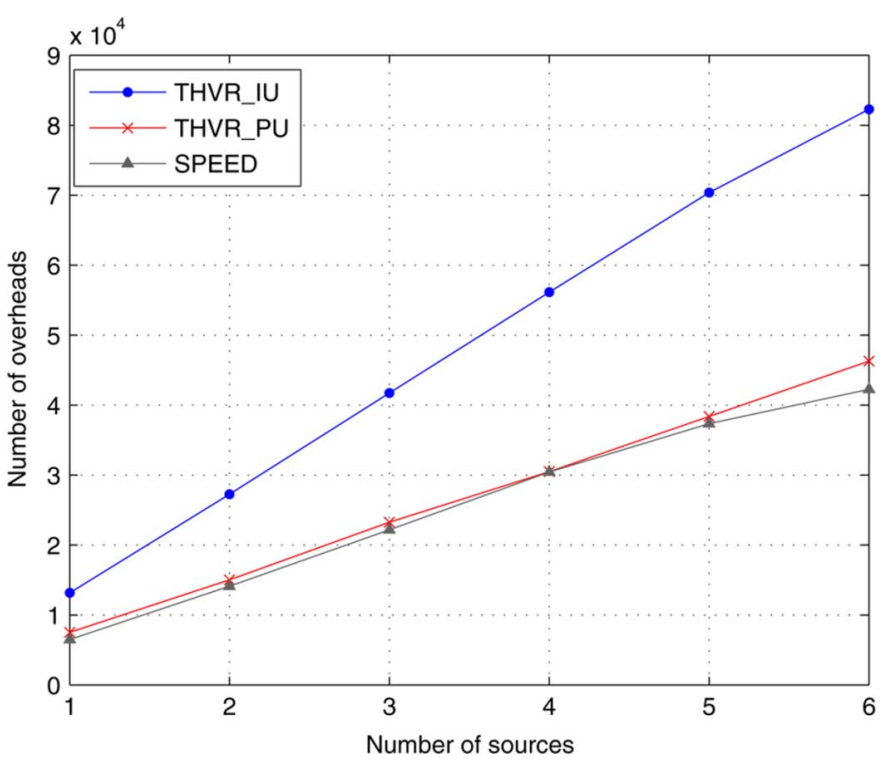

Fig. 9. Comparison of the number of overheads encountered in SPEED and THVR with instant update (IU) and with piggybacked update (PU).

times of that by SPEED. ${ }^{2}$ However, one can consider to reduce the overheads by piggybacking the updated information in conventional ACK packets but without further (extra) feedbacks. Consequently, these data will be piggybacked and sent together only when ACK is to be transmitted. This helps to maintain in a small number of feedback packets despite the fact that the resulting ACK size will be larger. By this approach, simulation shows that the number of overheads encountered in the WSN is almost the same as that in SPEED. Theoretically, they are in the same amount. The slight difference is due to simulation randomness. The result is plotted in Fig. 9 and labeled by "THVR_PU". Note that a drawback of this piggyback solution is that the two-hop delay information may not be updated frequently enough. However, since the link delay estimation is based on the combination of the historical average and most recent one, there could be only minor difference to the estimation performance even when the update is not immediate and especially in WSN with low mobility.

\section{CONCLUSION}

In this paper, a two-hop neighborhood information-based geographic routing protocol is proposed to enhance the service quality of real-time packet delivery for WSN. We adopt the approach of mapping packet deadline to the velocity as SPEED; however, the routing decision is made based on the two-hop

\footnotetext{
${ }^{2}$ Note that in SPEED, two methods can be used for updating neighborhood information: (i) periodic beacon announcement, or (ii) ACK piggyback. Here, we adopt the latter, by which update is embedded in ACK message.
} 
velocity integrated with energy balancing mechanism. An energy-efficient packet drop control is incorporated to enhance energy utilization efficiency while keeping low packet deadline miss ratio. The actual characteristics of physical and MAC layers are captured in the simulation studies. Simulation results show that, compared with SPEED and the $(\mathrm{PRR} \times d)$-routing which both only utilize one-hop information, THVR has achieved lower end-to-end deadline miss ratio and higher energy utilization efficiency.

\section{REFERENCES}

[1] J. M. Kahn, R. H. Katz, and K. S. J. Pister, "Next century challenges: Mobile networking for smart dust," in Proc. IEEE/ACM MobiCom, Aug. 1999, pp. 271-278.

[2] A. Willig, "Recent and emerging topics in wireless industrial communications: A selection," IEEE Trans. Ind. Infomat., vol. 4, no. 2, pp. 102-124, May 2008.

[3] Y. Li, C. S. Chen, Y.-Q. Song, and Z. Wang, "Real-time QoS support in wireless sensor networks: A survey," in Proc. IFAC FET, Nov. 2007, pp. 373-380.

[4] J. Stankovic, T. Abdelzaher, C. Lu, L. Sha, and J. Hou, "Real-time communication and coordination in embedded sensor networks," Proc. IEEE, vol. 91, no. 7, pp. 1002-1022, 2003.

[5] Y. Li, C. S. Chen, Y.-Q. Song, Z. Wang, and Y. Sun, "A two-hop based real-time routing protocol protocol for wireless sensor networks," in Proc. IEEE WFCS, May 2008, pp. 65-74.

[6] K. S. Prabh and T. F. Abdelzaher, "On scheduling and real-time capacity of hexagonal wireless sensor networks," in Proc. ECRTS, 2007, pp. 136-145.

[7] C. S. Chen, Y. Li, and Y.-Q. Song, "An exploration of geographic routing with $k$-hop based searching in wireless sensor networks," in Proc. CHINACOM, Aug. 2008, pp. 376-381.

[8] M. A. Spohn and J. J. Garcia-Luna-Aceves, "Enhancing broadcast operations in ad hoc networks with two-hop connected dominating sets," in Proc. IEEE MASS, 2004, pp. 543-545.

[9] W. Lou and J. Wu, "On reducing broadcast redundancy in ad hoc wireless networks," IEEE Trans. Mobile Comput., vol. 1, no. 2, pp. 111-122, 2002.

[10] V. Rajendran, K. Obraczka1, and J. J. Garcia-Luna-Aceves, "Energyefficient, collision-free medium access control for wireless sensor networks," Wireless Networks, vol. 12, no. 1, pp. 63-78, Feb. 2006.

[11] G. Calinescu, "Computing 2-hop neighborhoods in ad hoc wireless networks," in Proc. AdHocNow, 2003, pp. 175-186.

[12] T. He, J. Stankovic, C. Lu, and T. Abdelzaher, "A spatiotemporal communication protocol for wireless sensor networks," IEEE Trans. Parallel Distrib. Syst., vol. 16, no. 10, pp. 995-1006, 2005.

[13] Crossbow Motes, [Online]. Available: http://www.xbow.com

[14] J. Polastre, J. Hill, and D. Culler, "Versatile low power media access for wireless sensor networks," in Proc. ACM SenSys, 2004, pp. 95-107.

[15] B. Nefzi and Y.-Q. Song, "Performance analysis and improvement of Zigbee routing protocol," in Proc. IFAC FET, Nov. 2007, pp. 199-206.

[16] K. Akkaya and M. Younis, "An energy-aware QoS routing protocol for wireless sensor networks," in Proc. ICDCS, May 2003, pp. 710-715.

[17] N. Boughanmi and Y.-Q. Song, "A new routing metric for satisfying both energy and delay constraints in wireless sensor networks," $J$. Signal Process. Syst., vol. 51, no. 2, pp. 137-143, 2008.

[18] I. Stojmenovic, Handbook of Sensor Networks: Algorithms and Architectures. New York: Wiley, 2005

[19] Part 15.4: Wireless Medium Access Control (MAC) and Physical Layer (PHY) Specifications for Low-Rate Wireless Personal Area Networks (LR-WPANs), IEEE P802.15.4aD/4, Jul. 2006, (Amendment of IEEE Standard 802.15.4), IEEE-SA.

[20] E. Felemban, C. G. Lee, and E. Ekici, "MMSPEED: Multipath multispeed protocol for QoS guarantee of reliability and timeliness in wireless sensor network," IEEE Trans. Mobile Comput., vol. 5, no. 6, pp. 738-754, 2006.

[21] O. Chipara, Z. He, G. Xing, Q. Chen, X. Wang, C. Lu, J. Stankovic, and T. Abdelzaher, "Real-time power-aware routing in sensor network," in Proc. IWQoS, Jun. 2006, pp. 83-92.

[22] L. Bao and J. Garcia-Luna-Aceves, "Transmission scheduling in ad hoc networks with directional antennas," in Proc. MobiCom, 2002, pp. $48-58$.
[23] A. Woo, T. Tong, and D. Culler, "Taming the underlying challenges of reliable multihop routing in sensor networks," in Proc. ACM Sensys, Nov. 2003, pp. 14-27.

[24] Prowler, [Online]. Available: http://www.isis.vanderbilt.edu/projects/ nest/prowler

[25] Rmase, [Online]. Available: http://www2.parc.com/isl/groups/era/ nest/Rmase

[26] Y. Li, Y.-Q. Song, R. Schott, Z. Wang, and Y. Sun, "Impact of link unreliability and asymmetry on the quality of connectivity in largescale sensor networks," Sensors, vol. 8, no. 10, pp. 6674-6691, 2008.

[27] M. Zuniga and B. Krishnamachari, "Analyzing the transitional region in low power wireless links," in Proc. IEEE SECON, 2004, pp. $517-526$.

[28] K. Seada, M. Zuniga, A. Helmy, and B. Krishnamachari, "Energy-efficient forwarding strategies for geographic routing in lossy wireless sensor networks," in Proc. ACM SenSys, 2004, pp. 108-121.

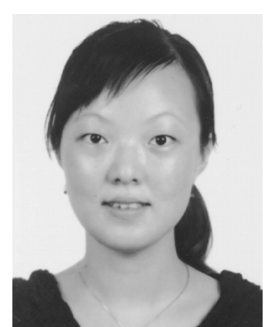

Yanjun Li received the B.S. degree from Zhejiang University, Hangzhou, China, in 2004. She then joined a successive postgraduate and doctoral program in control science and engineering at Zhejiang University. She is currently working towards the $\mathrm{Ph} . \mathrm{D}$. degree at Zhejiang University, Zhejiang, China, and the University of Henri Poincaré Nancy 1, France.

From 2007 to 2008, she was a visiting student in the Lorraine Laboratory of IT Research and Applications (LORIA), France. Her research interests include coverage, connectivity, routing and real-time QoS support for wireless sensor networks.

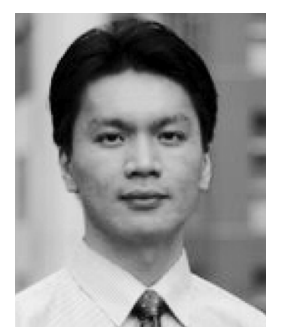

Chung Shue Chen (S'02-M'05) received the B.Eng., M.Phil., and Ph.D. degrees in information engineering from the Chinese University of Hong Kong, Shatin, Hong Kong, in 1999, 2001, and 2005, respectively.

From 2005 to 2006, he was an Assistant Professor at the Chinese University of Hong Kong. From 2006 to 2007, he was a Postdoctoral Researcher at the Centre National de la Recherche Scientifique (CNRS), Lorraine Laboratory of IT Research and Applications (LORIA), France. In 2007, he was awarded ERCIM "Alain Bensoussan" Fellowship and worked at the Norwegian University of Science and Technology (NTNU), Norway. Under the ERCIM Fellowship, in 2008, he was with Centrum Wiskunde and Informatica (CWI), The Netherlands. His research interests include radio resource allocation, multiple access control, and QoS management for wireless communication systems.

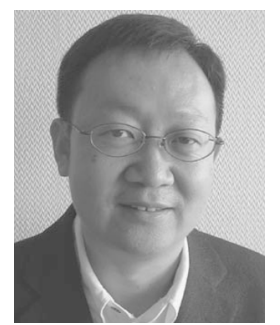

Ye-Qiong Song received the B.S. degree from Beijing University of Posts and Telecommunications, Beijing, China, in 1984, the M.S.E. degree from the Ecole National Supérieure des Télécommunications, Paris, France, in 1987, the M.Sc. degree (DEA) from the University of Paris 6, Paris, France, in 1988, the Ph.D. degree from the Institut National Polytechnique de Lorraine (INPL), Nancy, France, in 1991, and the Habilitation to Lead Research (HdR) degree from the University of Henri Poincaré Nancy 1 , in 2004, all in telecommunications and computer

science.

In 1988, he joined Lorraine Laboratory of IT Research and Applications (LORIA) Vandoeuvre-lès-Nancy, France. From 1992 to 2005, he was an Assistant Professor and then Associate Professor with the University of Henri Poincaré Nancy 1. From 2001 to 2003, he was a Full-Time Researcher with INRIA Lorraine. He is currently a Professor of Computer Science with INPL. His research interests include modeling and performance evaluation of networks and real-time distributed systems including networked control systems, and the implementation of real-time quality-of-service mechanisms in industrial networks, in-vehicle networks, IP networks, power line communication networks, and wireless sensor networks. 


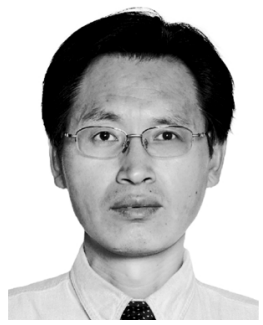

Zhi Wang (M'08) received the B.S. degree from Shenyang Jian Zhu University, Shenyang, China, in 1991, the M.S. degree from Southeast University, China, in 1997, and the Ph.D. degree from Shenyang Institute of Automation, the Chinese Academy of Sciences, Shenyang, China, in 2000.

From 2001 to 2002, he was a Postdoctoral Researcher at the Lorraine Laboratory of IT Research and Applications (LORIA), France. From 2007 to 2008, he was a Visiting Researcher with Jönköping University and the Royal Institute of Technology, Sweden. He is currently an Associated Professor of Control Science and Engineering with Zhejiang University. His research interests include mobile sensing systems including agent-based information processing, real-time classification and tracking; industrial communication and systems including reliable and real-time communication protocols and networked control systems.

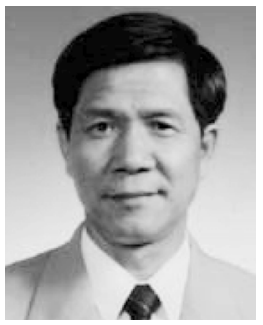

Youxian Sun graduated from the Department of Chemical Engineering, Zhejiang University, Zhejiang, China, in 1959.

He was an Associate Professor in 1982 and Professor in 1988 with Zhejiang University. From 1984 to 1987 , he was a Visiting Professor with a Humboldt Fellowship at Stuttgart University, Germany. His research interests include control and optimization of complex engineering systems, robust control theory and applications, theory and methodologies for industrial process integrated automation systems, complex networks, and wireless sensor networks.

Prof. Sun was elected as a Member of the Chinese Academy of Engineering in 1995. 